\title{
Sur l'économie politique de la réglementation
}

\section{Jean-Jacques Laffont}

\section{Résumé}

Les théoriciens ont au cours de la dernière décennie mis au point les fondements d'une réglementation normative optimale. Se fondant sur l'exemple de la tarification d'une voie de communication, l'article montre qu'il faut aller plus loin et rechercher un arbitrage entre deux maux : l'inefficacité économique et l'arbitraire politique. Les modes tarifaires sophistiqués imaginés par la théorie laissent en effet plus de liberté au politique de les détourner au profit de ses intérêts propres ou de ceux du groupe qui le soutient. C'est un arbitrage identique qui est à prendre en compte pour fixer les règles constitutionnelles gouvernant la réglementation et les privatisations.

\begin{abstract}
During the past decade theoreticians have laid the foundations of optimum normative regulation. Taking as an example the pricing of a communication channel, the article shows the need to go even further and to choose the lesser of two evils: economic inefficiency or political arbitrariness. In theory, sophisticated modes of pricing grant considerable latitude to policy makers to divert them in their own interests or in those of the group supporting them. Exactly the same type of choice has to be made in establishing constitutional rules governing regulation and privatization.
\end{abstract}

\section{Citer ce document / Cite this document :}

Laffont Jean-Jacques. Sur l'économie politique de la réglementation. In: Réseaux, volume 13, n72-73, 1995. L'économie des télécommunications. pp. 11-18;

doi : 10.3406/reso.1995.2709

http://www.persee.fr/doc/reso_0751-7971_1995_num_13_72_2709

Document généré le 07/06/2016 


\section{SUR L'ÉCONOMIE POLITIQUE DE LA RÉGLEMENTATION}

Jean-Jacques LAFFONT 


$$
-12
$$


$\mathrm{P}$ renant appui sur les progrès de la théorie des jeux, de l'économie de l'information et de l'économie des incitations des années soixante-dix, les théoriciens de l'économie ont élaboré dans les années quatre-vingt un cadre normatif qui a permis d'enrichir considérablement les débats sur la réglementation des monopoles naturels et en particulier des réseaux d'infrastructure, comme ceux des télécommunications.

En mettant l'accent sur le problème fondamental des difficultés informationnelles des régulateurs - que ce soit au niveau de la connaissance de la demande et surtout au niveau de la connaissance des coûts - la théorie a permis de préciser un arbitrage essentiel entre l'abandon de rentes informationnelles socialement coûteuses à certains acteurs économiques et les distorsions d'efficacité qui permettent d'atténuer ces rentes. Ainsi, on a pu comprendre la tension entre des réglementations contrôlant le taux de rendement des actifs investis (rate of return regulation) qui privilégient l'extraction des rentes en sacrifiant la minimisation des coûts, et des réglementations fondées sur un plafond de prix (price cap regulation) qui privilégient l'efficacité aux dépens de l'abandon d'importantes rentes informationnelles.

La réglementation normative optimale apparaît alors comme la combinaison de schémas incitatifs (plus on rembourse les coûts moins on incite à l'effort de minimisation des coûts) et de tarification à la Ramsey-Boiteux assise sur les coûts marginaux et les élasticités (1).

Cette théorie a eu le mérite de clarifier nombre de discussions et de sortir l'économie de la réglementation d'un état que l'on pourrait qualifier de préscientifique eu égard aux progrès de la science économique. Il est toutefois frappant de voir la résistance des milieux concernés vis-à-vis des conclusions obtenues, en particulier des milieux juridiques qui élaborent les cadres régulatoires européens. Les critiques soulignent la complexité des règles proposées par les économistes, l'interdiction souvent effective de l'utilisation de transferts compensatoires entre régulateurs et entreprises régulées, la complexité de l'évaluation des élasticités prix.

Une partie de ces critiques relèvent de la non-compréhension pure et simple, de la frilosité des décideurs face à des innovations qu'ils ne maîtrisent pas. Toutefois, je pense qu'une partie des résistances vient du manque d'attention des économistes aux contraintes politiques. Pour passer de la théorie à la politique économique, l'incorporation de ces contraintes dans une économie politique de la réglementation est nécessaire. L'objectif de cette note est triple. Dans une première section, nous essayerons d'expliquer pourquoi, en économie industrielle, la théorie économique a pu être tentée de ne pas intégrer ces contraintes et comment la décentralisation de l'information et les contraintes incitatives modifient les relations entre économistes et décideurs politiques et conduisent les économistes à une nécessaire économie politique. Dans une deuxième section, nous prendrons un exemple simple, celui du choix de la tarification d'une voie de communication, pour montrer que les économistes classiques raison- 
naient dans ce cadre élargi de l'économie politique et comment la théorie moderne peut tenter de progresser dans ce domaine. Enfin, dans une dernière partie nous élargirons l'analyse pour envisager d'un point de vue d'économie politique les questions plus vastes de la privatisation et de la réglementation.

Les réflexions que nous rassemblons dans cet article sont préliminaires et reposent sur des modèles théoriques qu'il sera nécessaire d'affiner (2).

\section{Information ef relation entre économistes ef politiques}

En 1970, lors de la conférence qu'il donna à l'occasion de son prix Nobel (le premier prix Nobel, qu'il partagera avec Tinbergen), Ragnar Frisch a pu dire :

«Ce n'est pas la tâche des économètres et des ingénieurs sociaux d'entrer dans une discussion détaillée du système politique. "

Le seul souci de Frisch était de dialoguer avec les politiciens pour déterminer leurs préférences et ensuite les aider à définir les politiques économiques qui leur permettraient d'optimiser leurs choix au regard de ces préférences.

Les difficultés qu'impliquent cette attitude face au problème de la distribution des revenus ont parfois été soulignées. Prenons toutefois le cas le plus favorable dans lequel on ignore les effets revenus. Dans un monde d'information complète, la prise de pouvoir par le jeu démocratique peut conduire à privilégier certaines catégories mais, dans la mesure où les transferts de revenus entre agents économiques se font sans perte d'efficacité, on peut comprendre que les alternances permises par le jeu démocratique rassurent l'économiste au service des décideurs sociaux. En effet, en moyenne, l'allocation des ressources sera efficace et, si les contraintes constitutionnelles limitent les redistributions de façon raisonnable, on pourra négliger le manque d'assurance qu'implique le jeu démocratique (on n'assure pas le risque démocratique).

Il en est tout autrement lorsqu' on prend en compte la décentralisation de l'information et le comportement stratégique des acteurs économiques vis-à-vis de leur information privée. C'est ainsi que la littérature sur l'impôt sur les revenus, à partir des travaux d'Edgeworth, Vickrey et Mirrlees, a bien expliqué que les contraintes incitatives rendent coûteuses les redistributions de revenus. Empiriquement, on peut les résumer en disant que pour donner 1 franc à une catégorie sociale il en coûte de 1,3 à 2 voire 3 francs à la société selon la qualité du système fiscal considéré. Techniquement cela veut dire qu'on ne se déplace plus sur une frontière de Pareto linéaire mais au contraire très convexifiée. Il en résulte que les maximisations par les majorités successives de leurs intérêts propres conduisent en moyenne à des niveaux d'utilité très en deçà de ce qui résulterait de la maximisation d'un bien-être social défini une fois pour toute.

L'opportunité dès lors existe de propositions de politique économique qui d'un point de vue strictement économique peuvent apparaître fort inefficaces, mais qui limitent tellement bien le niveau de discrétion des décideurs politiques, qu'en moyenne elles conduisent à une amélioration du bien-être social. C'est ainsi qu'il faut comprendre aujourd'hui le débat américain sur la contrainte constitutionnelle de l'équilibre budgétaire annuel imposé aux politiques ou le débat émergeant en France sur l'opportunité d'une règle constitutionnelle qui empêcherait les nationalisations et privatisations successives.

L'exemple suivant va tenter d'illustrer cette problématique. Avant de le développer, notons que la situation peut être encore aggravée si on soupçonne les politiciens de viser des objectifs privés ; l'expert, en privant le politique d'information, peut alors limiter la réalisation par celui-ci de ses objectifs privés et par suite, améliorer le bien-être social. Dans cette vision, le rôle de l'économiste se modifie et 
s'élargit. Sa fonction de conseil du politique est à redéfinir ; d'un autre côté il peut proposer des règles économiques constitutionnelles qui améliorent le bienêtre social en moyenne en limitant de façon appropriée la discrétion des politiciens.

\section{Sur la tarification des monopoles naturels}

Prenons l'exemple simple d'une voie de communication, route, canal, pont ou transport ferroviaire, voire réseau optique.

La théorie économique pure suggère la tarification au coût marginal et le financement des coûts fixes par l'Etat. La prise en compte du coût social des fonds publics conduit à la tarification Ramsey-Boiteux qui fait contribuer le service considéré au financement des coûts fixes d'une façon inversement proportionnelle à l'élasticitéprix de la demande du bien en question. La prise en compte d'asymétries d'information sur les coûts peut conduire à des corrections incitatives supplémentaires pour limiter les rentes informationnelles. Voilà en quelques mots l'état de la question.

La pratique courante est pourtant de renoncer à la prise en compte des élasticités prix et de se contenter de gonfler uniformément les prix au-delà des coûts marginaux pour parvenir à l'équilibre budgétaire. C'est ainsi que les tarifs de l'électricité ont été conçus, selon une doctrine que l'on appelle en France la règle d'Allais.

Selon la théorie, une telle tarification semble bien inefficace puisqu'on distord les prix jusqu'à couvrir tous les coûts (ce qui peut conduire à de larges distorsions si les coûts fixes sont importants) et qu'on renonce à faire supporter les surtarifications en priorité aux biens dont la demande est la plus inélastique. Les quantités consommées sont alors très éloignées de l'optimum.
Le retour aux économistes classiques nous donne pourtant une clé d'interprétation.

Adam Smith (3) est particulièrement clair sur ce point.

«Il ne semble pas nécessaire que les dépenses de ces projets publics soient couvertes par le budget de l'Etat...»

Discutant des routes, il énonce que les utilisateurs doivent payer en proportion du coût marginal qu'ils imposent aux infrastructures, ce qui nous amène donc à la règle d'Allais.

La motivation essentielle d'Adam Smith est politique.

"Une magnifique route ne peut être construite dans un désert où il n'y a pas ou peu de commerce, ou simplement parce qu'elle conduit au domaine d'un intendant de province, ou d'un grand lord que l'intendant veut courtiser. Un grand pont ne peut être jeté au-dessus d'une rivière à un endroit où personne ne passe, simplement pour embellir la vue découverte à partir d'un palais voisin. »

Adam Smith est conscient que les économistes n'ont pas le pouvoir et que la mise en œuvre d'une règle de tarification sera déléguée à des intermédiaires, politiques ou non, qui auront une grande marge de discrétion sur certains éléments de la règle. Ainsi, dans son exemple, en trichant sur la réalité des coûts fixes, une route socialement inefficace pourra être construite pour satisfaire un groupe d'intérêts en la finançant sur fonds publics. Walras (4) suivra essentiellement le point de vue de Smith en notant l'intérêt qu'il y a toutefois à subventionner le projet pour les effets externes positifs qu'il peut procurer. Quand Edgeworth (5) s'interrogera à la suite de Dupuit (6) sur la tarification des trains, il fera appel à la discrimination par les prix pour assurer l'équilibre budgétaire en renonçant ainsi moins souvent à des projets socialement valables mais dont les coûts ne peuvent être couverts par des tarifs uniformes. 
Il faudra attendre des socialistes qui prônaient la tarification au coût marginal pour justifier la nationalisation (7), ou des théoriciens qui pensaient que la société peut fonctionner à la lumière de la rationalité économique pour prôner la tarification au coût marginal et l'analyse de surplus. Ainsi Hotelling (8) affirme :

"Cette proposition a des implications révolutionnaires, par exemple pour l'industrie électrique et l'économie des transports ferroviaires, en montrant que la société devrait diminuer considérablement les tarifs et remplacer les revenus perdus par des subventions. »

Pour comprendre pourquoi la rationalité économique peut avoir des effets pervers, il faut se reporter au cadre constitutionnel dans lequel évoluent les hommes politiques. La constitution est très simple, les pouvoirs judiciaires limités, et donc un contrat complet ne peut régir le fonctionnement des politiques. La délégation des décisions aux hommes politiques s'accompagne de larges plages discrétionnaires. Comme ceux-ci ont des objectifs privés ou réagissent aux intérêts des groupes qui les ont élus, ils sont conduits à des politiques de redistribution excessivement coûteuses, qu'elles soient à leur profit ou à celui du groupe qui les soutient.

A titre d'exemple, comparons deux règles qui ont des implications différentes sur l'arbitrage entre efficacité et discrétion évoqué ci-dessus.

Comme nous l'avons mentionné, la règle de Smith est inefficace d'un point de vue strictement économique. Toutefois, elle ne laisse aucune marge de manœuvre au politique.

Considérons maintenant le cas où il existe deux catégories de consommateurs. Une règle plus efficace a priori consiste à fixer un prix différent pour chaque catégorie de façon à couvrir les coûts. Mais, comme chaque majorité choisira son tarif, elle sera conduite à une discrimination soit excessive, soit insuffisante en faveur de l'une ou l'autre catégorie de consomma- teurs. Ainsi cette règle qui, appliquée par un régulateur bienveillant améliorerait le bien-être social par rapport à la règle rigide de Smith, accroît la discrétion des politiques. Si les coûts fixes ne sont pas trop importants (ce qui limite les inefficacités de la règle de Smith), et si la différenciation des consommateurs est grande (ce qui accroît les coûts de la discrimination politique), cette nouvelle règle est moins bonne en moyenne que la règle de Smith. Au contraire, si les coûts fixes sont importants et la différenciation faible, les distorsions de la discrimination politique sont moins coûteuses socialement que les grandes distorsions uniformes impliquées par la règle de Smith.

Avec une modélisation appropriée du jeu politique considéré, on peut ainsi comparer les règles constitutionnelles qui fixent les formes tarifaires en arbitrant entre deux maux : inefficacité économique et discrétion politique.

\section{Vers une économie politique de l'économie industrielle}

Le même cadre d'analyse peut être étendu pour discuter du problème de la privatisation ou de la structuration d'un cadre institutionnel pour la réglementation.

Supposons par exemple que la faiblesse des contraintes constitutionnelles permette à une majorité politique de s'approprier les rentes informationnelles qu'offre le management d'entreprises publiques. La propriété publique permet alors à chaque majorité, à son tour, de $s$ 'approprier ces rentes, en choisissant une régulation qui favorise des rentes importantes malgré leurs coûts sociaux évoqués plus haut. La propriété privée permet au contraire aux propriétaires de monopoles privés de préserver leurs rentes ; les majorités politiques qui ont peu de relations avec ces propriétaires choisiront alors des régulations trop défavorables aux rentes et accepteront des in- 
efficacités excessives dans la minimisation des coûts.

Les contraintes politiques redonnent donc un rôle important aux droits de propriété ; en outre, l'arbitrage entre propriété publique et propriété privée, dépend ici de l'importance des asymétries d'information et de la taille des majorités en particulier.

Si maintenant, on souhaite mettre en place des institutions de réglementation qui limitent par leur action l'importance des asymétries d'information, on comprend que les opportunités de capture de ces institutions seront différentes, dans le cas de la propriété privée, selon le type de majorité. Ces institutions seront inutiles quand le pouvoir politique fera collusion avec les propriétaires des entreprises ; au contraire lorsque le pouvoir politique opposé à ces groupes d'intérêt cherchera à obtenir par le biais de l'agence de régulation unc limitation de leurs rentes, les incitations à la capture de celle-ci par les intérêts privés seront fortes.

Les choix constitutionnels qui garantiront plus ou moins d'indépendance aux commissions de régulation résulteront donc d'arbitrages entre les inconvénients de leur capture potentielle et les coûts de leur indépendance.

\section{En guise de conclusion}

La science politique nous a montré comment la structuration du pouvoir, l'établissement de contre-pouvoirs offre plus ou moins de prise aux possibilités de capture par les intérêts privés et plus ou moins de discrétion aux décideurs politiques. Puisque la prise en compte de l'information privée démultiplie les distorsions économiques de la discrétion politique, contrairement à ce que pensait R. Frisch, seule la discussion détaillée du système politique peut nous conduire du monde de la rationalité économique à celui de la rationalité sociale.

Bien sûr, il faut espérer que l'approfondissement de la démocratie, la sophistication des institutions politiques et judiciaires permettra de rapprocher l'économie politique de la théorie économique pure. En attendant, il est urgent, pour que les messages importants de la rationalité économique soient compris et fassent leur chemin dans la pratique sociale, que les économistes sachent se garder dans leurs propositions de politique économique des dangers les plus importants engendrés par la nécessaire délégation de leur mise en œuvre à des hommes politiques, agissant selon leurs objectifs ou leurs contraintes propres. 
BARON D. and MYERSON R., " Regulating a Monopolist with Unknown Costs », Econometrica, 50, 911-930, 1982.

DUPUIT J., « De l'influence des péages sur l'utilité des voies de communication », Annales des ponts et chaussées, 1849.

EDGEWORTH B,. « Contributions to the Theory of Railways », Economic Journal, 1913.

FINSINGER J. and VOGELSANG I., «Regulatory Adjustement Process for Optimal Pricing by Multiproduct Monopoly Firms ", Bell Journal of Economics, 10 , 157-171, 1979.

HOTELLING H., " The General Welfare in Relation to Problems of Taxation and of Railway and Utility Rates ", Econometrica, 242-269, 1939.

LAFFONT J.-J., « Industrial Policy and Politics », International Journal of Industrial Organization, 1995.
LAFFONT J.-J., « Frisch, Hotelling and the Marginal Cost Pricing Controversy ", Centenial of R. Frisch, Oslo, 1995.

LAFFONT J.-J. and TIROLE J., $A$ Theory of Incentives in Procurement and Regulation, MIT Press, Cambridge, 1993.

LAUNDHART W, Mathematische Begründung der Volkswitschaftslehre, 1885.

LOEB M. and MAGAT W., «A Decentralized Method of Utility Regulation», Journal of Law and Economics, 22, 399404, 1979.

SMITH A., The Wealth of Nations, The Modern Library, New York (1937 edition), 1776.

WALRAS L., " L'Etat et les chemins de fer ", Revue du droit public et de la science politique, mai-juin et juillet-août, reproduit dans August et Leon Walras CEuvres Economiques Complètes Vol. X, Economica, 1992, Paris, 1897. 